\title{
Multizone Adaptive Grid Generation Technique for Multilayer Multistep Process Simulation
}

\author{
M. K. Moallemi and H. Zhang \\ Department of Mechanical Engineering, Polytechnic University \\ 6 Metrotech Center, Brooklyn, NY 11201, USA
}

\begin{abstract}
A multizone adaptive grid generation technique is developed and used with a curvilinear finite-volume approach to simulate multistep IC processes on non-planar multilayer structures with moving boundaries. The capabilities of the numerical scheme is demonstrated by simulating silicon oxidation and impurity diffusion in trench structures with corner angles equal to, and greater than $90^{\circ}$.
\end{abstract}

\section{Introduction}

An IC process simulator, as a design and development tool for today's VLSI technology, must be able to model multistep processes on multilayer nonplanar structures which may also involve moving boundaries. Several two- and three-dimensional [1-3] process simulators have been reported in the literature, each with its own merits and demerits. The principal shortcomings of these simulators are $a$ ) their inability to handle generalized geometries (e.g., structures with corner angles $\left.\geq 90^{\circ}\right)$, and $b$ ) their inaccurate or inefficient treatments of moving boundaries or irregular domains. These deficiencies are mostly due to the simplistic approach to the discretization of the problem domain and/or the model equations [1-4]. Recent trend in the evolution of process simulators has been towards the use of numerical grid generating techniques with desirable characteristics such as boundary-fitting capability [3-4], and adaptivity to the solution development [4-5]. These grid generating routines provide geometric flexibility, but require special consideration in the discretization of the governing equations. Simple analytical transformation of the equations to the computational domain is known to fail in simulation of processes in structures with corner angle $\geq 90^{\circ}$.

In this paper, a numerical methodology is presented that utilizes a multizone adaptive grid generation technique [5] for the discretization of the physical domain, and a curvilinear finite-volume approach [5] for the discretization of the governing equations (in the physical space) and development of finite difference equations. The numerical scheme is validated by simulating silicon oxidation process (with the inclusion of stress effects) on a $50^{\circ}$ trench and comparing the results with the published SEM photographs [6]. The capabilities of this numerical method is demonstrated by simulating oxidation of silicon on a $90^{\circ}$ trench and an undercut structure. 


\section{Physical Model}

Simultaneous and sequential silicon oxidation and impurity diffusion in nonplanar structures are modeled. The oxidation model is based on a steady state oxidant diffusion, and a slow incompressible viscous flow of oxide [7]. The effects of oxidation-induced stress on the oxide growth which are significant in the nonplanar structures are accounted for by the use of stress-dependent physical parameters [6]. Impurity diffusion in the silicon and oxide is assumed to be transient, and nonlinear effects are considered by taking the effective diffusion coefficient to be function of concentration [8]. Under oxidizing conditions, the impurity segregation, moving boundary flux, and oxidation-enhanced diffusion effects are also included in the model. The simulations presented here were performed with the input physical parameters used in [6] and [8] to permit comparison. The details of the physical model and the values of the input parameters are provided in [5].

\section{Numerical Method}

The major building block of the numerical method is a multizone adaptive grid generating technique. The original technique was developed by Brackbill and Saltzman [9] who used the variational method to minimizing a linear combination of integrals which are measures of different grid characteristics, including smoothness, orthogonality and weighted cell area of the grids. To add the multizoning feature, this technique was modified by using constrained variational minimization on the grid line that separates two zones, thus, permitting the grid nods to move along this interface line only. To improve the orthogonality of the grid system near the moving and free boundaries, as well as its adaptivity to the movement of the oxide interface, geometric and local weighting functions were also included [5].

Over this nonorthogonal grid system, the governing equations are discretized using a curvilinear finite-volume approach. This approach is based on flux discretization in the physical domain, therefore, circumvents the limitations of direct (analytical) transformation (i.e., large artificial source terms). More detailed information about the numerical methodology and its implementation are provided in [5].

\section{Results and Discussions}

The accuracy of the numerical scheme was validated by performing simulation of oxidation on a $50^{\circ}$ trench $(1 \mu \mathrm{m}$ deep and $3 \mu \mathrm{m}$ wide). As shown in Fig. 1 , the predictions of this simulation are in excellent agreement with the SEM data [6]. A second simulation was performed on a $90^{\circ}$ trench, with initial grid system and boron distribution of Fig. 2. The grid distribution, the trench shape, and boron redistribution after 40.0 minutes of oxidation in wet $\mathrm{O}_{2}$ at $1000{ }^{\circ} \mathrm{C}$ are presented in Fig. 3. The figure clearly indicates the effects of stress, surface curvature and orientation on oxide growth. A comparison between Figs. 2 and 3 reveals the evolution (adaptivity) of the grid system with time and growth of the oxide layer. Figure 3 shows a big drop in the boron concentration in the silicon in the vicinity of oxide interface that is attributed to the larger diffusion coefficient for boron in the oxide compared to the silicon substrate. The third simulation was performed on an asymmetrical trench structure with initial width of $1.8 \mu \mathrm{m}$ and corner angles $112^{\circ}$ and $90^{\circ}$. The cross section of the structure and the corresponding grid system after 7.2 minutes of oxidation in a wet ambient at $1000{ }^{\circ} \mathrm{C}$ is shown in Fig. 4. The figure indicates nonuniform oxide growth due to stress and orientation dependence of oxidation rate parameters. 


\section{Conclusion}

A numerical scheme was developed utilizing a multizone adaptive grid generation technique, and a curvilinear finite-volume approach. The capabilities of the method was demonstrated by simulating silicon oxidation and impurity diffusion in trench structures with different corner angles. The work proved that this methodology has potential for the development of a robust and versatile tool for IC process modeling.

\section{References}

[1] B. Baccus, D. Collard, E. Dubois, D. Morel, "IMPACT4 -- A general twodimensional multilayer simulator," in Proc. Inter. Conf. on Semiconductor Devices and Processes, G. Baccarani, and M. Rudan (Eds.), Vol. 3, pp. 255-266, 1988.

[2] M. E. Law, C. S. Rafferty, and R. W. Dutton, SUPREM-IV. Technical report integrated circuits laboratories, Stanford University, Stanford, CA, 1988.

[3] S. Odanaka, H. Umimoto, M. Wakabayashi, and H. Esaki, "SMART-P, Rigorous three-dimensional process simulator on a supercomputer," IEEE Trans. Computer Aided Design, Vol. 7, No. 6, pp. 675-683, 1988.

[4] K. Wimmer, R. Bauer, S. Halama, G. Hobler, and S. Selberherr, "Transformation methods for nonplanar process simulation," Simulation of Semiconductor Devices and Processes, Vol. 4, pp. 131-138, 1991.

[5] M. K. Moallemi, and H. Zhang, "Application of multizone adaptive grid generation technique for simulation of multistep IC processes in nonplanar structures", IEEE Trans. Computer Aided Design (submitted).

[6] H. Umimoto, S. Odanaka, I. Nakao, and H. Esaki, "Numerical modeling of nonplanar oxidation coupled with stress effects," IEEE Trans. Computer Aided Design, Vol. 38, No. 3, pp. 505-511, 1991.

[7] D. Chin, S. Y. Oh, S. M. Hu, R. W. Dutton, and J. L. Moll, "Two-dimensional oxidation," IEEE Trans. Electron Devices, Vol. ED-30, No. 7, pp. 744-749, 1983.

[8] D. Anderson, and K. O. Jeppson, "Nonlinear two-step diffusion in semiconductors," J. Electrochem. Soc., Vol. 131, No. 11, pp. 2675-2679, 1984.

[9] J. U. Brackbill, and J. S. Saltzman, "Adaptive zoning for singular problems in two dimensions," J. Comp. Phys., Vol. 46, pp. 342-368, 1982.
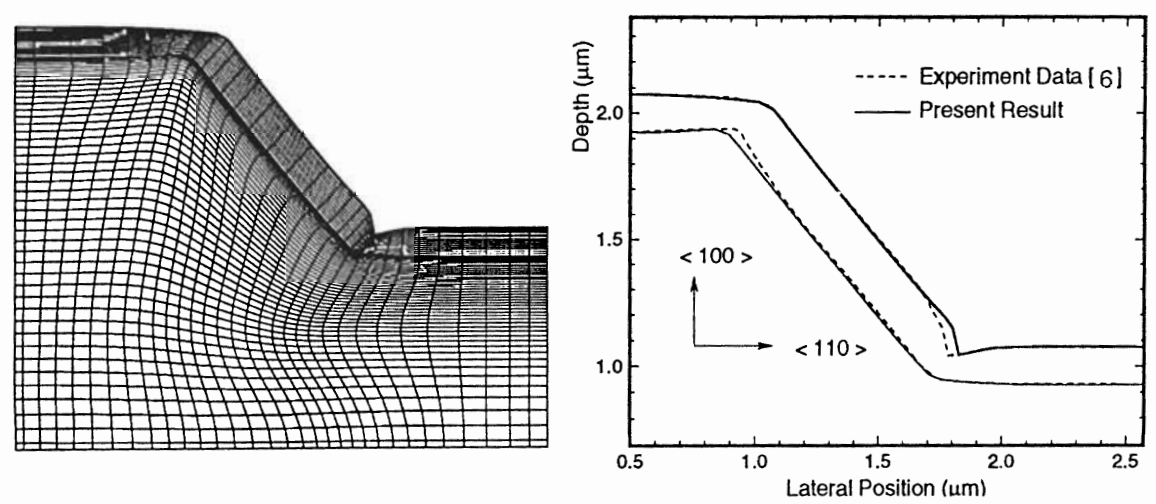

Fig. 1 Grid distribution and comparison of oxide profile in a trench after 20 minutes oxidation in a wet ambient at $1000{ }^{\circ} \mathrm{C}$. 

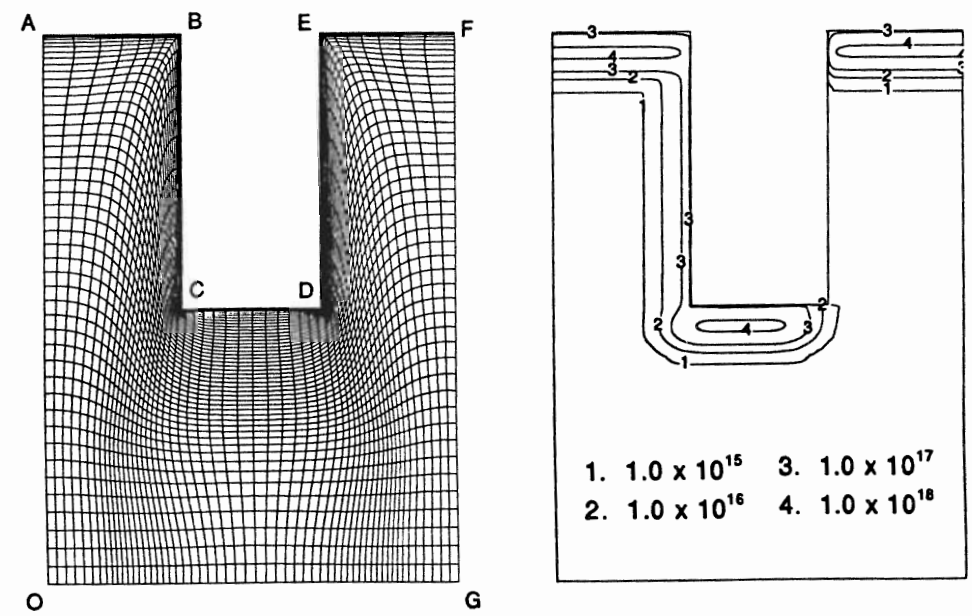

Fig. 2 Initial grid system and boron distribution $(O A=2.0 \mu \mathrm{m}, O G=1.5 \mu \mathrm{m})$.
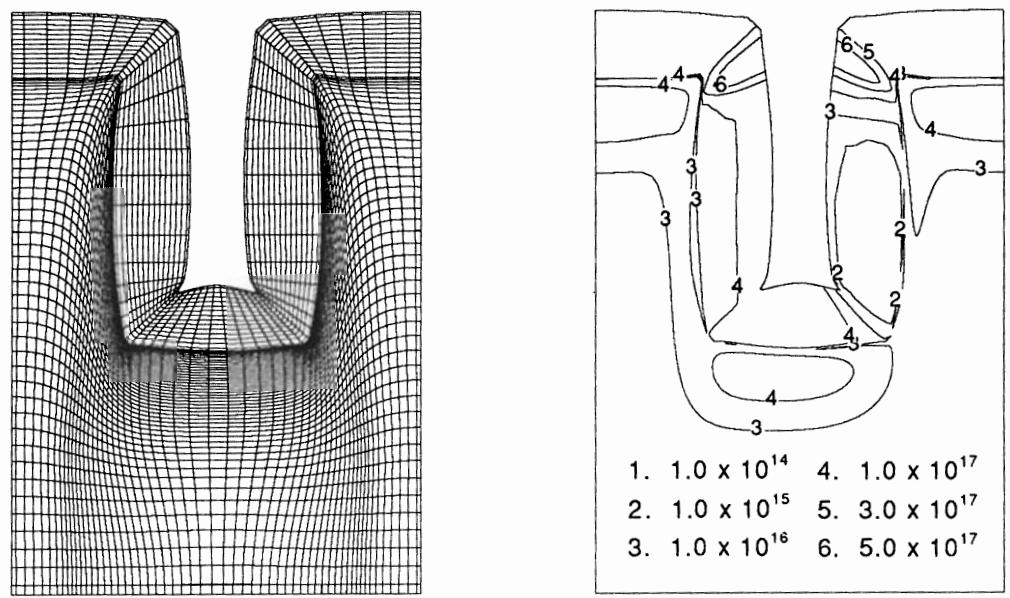

Fig. 3 Grid distribution, oxide profile and boron distribution after 40 minutes.

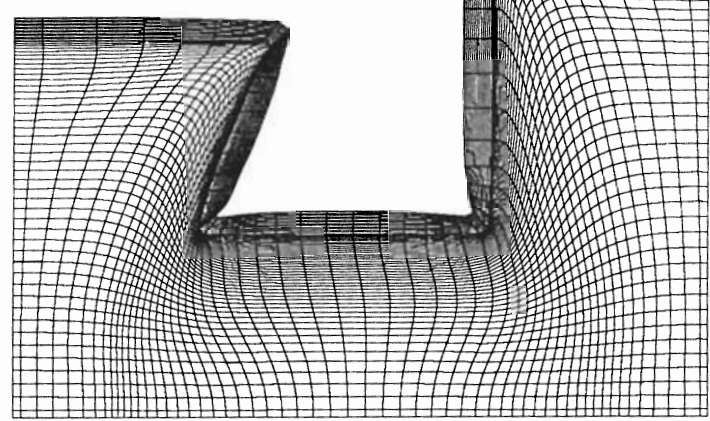

Fig. 4 Grid system and oxide profile after 7.2 minutes oxidation. 